\title{
Blow-up problems for a compressible reactive gas model
}

\author{
Zhengqiu Ling $^{1 *}$ and Zejia Wang ${ }^{2}$
}

\section{"Correspondence:} lingzq00@tom.com

${ }^{1}$ College of Mathematics and Information Science, Yulin Normal

University, Yulin, Guangxi 537000, P.R. China

Full list of author information is available at the end of the article

\begin{abstract}
This paper investigates a compressible reactive gas model with homogeneous Dirichlet boundary conditions. Under the parameters and the initial data satisfying some conditions, we prove that the solutions have global blow-up, and the blow-up rate is uniform in all compact subsets of the domain. Moreover, the blow-up rates of $|u(t)|_{\infty}$ and $|v(t)|_{\infty}$ are precisely determined.
\end{abstract}

MSC: 35K05; 35K55; 35D55

Keywords: degenerate parabolic system; nonlocal sources; finite time blow-up; blow-up rate

\section{Introduction and main results}

In this paper, we investigate blow-up and the blow-up rate of nonnegative solutions for the following degenerate reaction-diffusion system with nonlocal sources:

$$
\left\{\begin{array}{l}
u_{t}=\nabla \cdot\left(u^{m} \nabla u\right)+a u^{p_{1}}\|v\|_{B, \alpha_{1}}^{p_{2}}, \quad(x, t) \in B \times(0, T), \\
v_{t}=\nabla \cdot\left(v^{n} \nabla v\right)+b v^{q_{1}}\|u\|_{B, \alpha_{2}}^{q_{2}}, \quad(x, t) \in B \times(0, T), \\
u(x, 0)=u_{0}(x), \quad v(x, 0)=v_{0}(x), \quad x \in B, \\
u(x, t)=v(x, t)=0, \quad(x, t) \in \partial B \times(0, T),
\end{array}\right.
$$

where $B=B(0, R) \subset \mathbb{R}^{N}(N \geq 1)$ is a ball centered at the origin with the radius $R \in \mathbb{R}^{+}$, $a, b>0$, exponents $p_{2}, q_{2}, \alpha_{1}, \alpha_{2} \geq 1, m, n, p_{1}, q_{1}>0$, and $T<\infty$ is the maximal existence time of a solution, $\|\cdot\|_{B, \alpha}^{\alpha}=\int_{B}|\cdot|^{\alpha} \mathrm{d} x$.

The system (1.1) models such as heat propagations in a two-components combustible mixture gases [1]. This problem is worth studying because of the applications to heat and mass transport processes (see [2, 3]). In addition, there exist interesting interactions among the multi-nonlinearities described by these exponents in the problem (1.1).

In the past decades, many physical phenomena have been formulated into nonlocal mathematical models and studied by many authors. Here, we will recall some of those results concerning the first initial boundary value problem.

At first, the global solutions and blow-up problems for a single parabolic equation with nonlocal nonlinearity sources had been studied extensively, see [4-10] and references therein. As a typical example, in [4] Souplet considered the equation with spatial integral

(c) 2012 Ling and Wang; licensee Springer. This is an Open Access article distributed under the terms of the Creative Commons Attribution License (http://creativecommons.org/licenses/by/2.0), which permits unrestricted use, distribution, and reproduction in any medium, provided the original work is properly cited. 
term

$$
u_{t}=\Delta u+g\left(\int_{\Omega} f(u(x, t)) \mathrm{d} x\right)
$$

and the equation with both local and nonlocal terms

$$
u_{t}=\Delta u+\int_{\Omega} f(u(x, t)) \mathrm{d} x+h(u(x, t)) .
$$

These two equations are related to some ignition models for compressible reactive gases. The author introduced a method to investigate the profile of blow-up solutions of (1.2) and (1.3) and observed the asymptotic blow-up behaviors of the solutions. In addition, an important model in the theory of nuclear reactor dynamics can be described by the following equation with the space-time integral term:

$$
u_{t}=\Delta u+f\left(\int_{0}^{t} \int_{\Omega} g(u(y, s)) \beta(y) \mathrm{d} y \mathrm{~d} s\right) .
$$

The blow-up of its solutions was studied by Pao [5], Guo and $\mathrm{Su}$ [6].

In 2003, Li and Xie [7] considered the following problem:

$$
v_{\tau}=\Delta v^{m}+a v^{p_{1}} \int_{\Omega} v^{q_{1}} \mathrm{~d} x .
$$

By introducing some transformations $u=v^{m}, t=m \tau$ (1.5) takes the form

$$
u_{t}=u^{p}\left(\Delta u+a u^{r} \int_{\Omega} u^{s} \mathrm{~d} x\right)
$$

Then they proved that the solution of (1.6) blows up in finite time for large initial data and obtained the blow-up rate. Recently, Liu et al. in [8] investigated the blow-up rate of solutions to diffusion equation (1.6). Their approach was based on sub-and super-solution methods which were very different from those previously used in the study of the blow-up rate. They proved, by using the maximum principle, that the solutions have global blowup, and the rate of blow-up is uniform in all compact subsets of the domain. Here the global blow-up means that there exists $0<T<+\infty$ such that

$$
\lim _{t \rightarrow T^{-}}|u(\cdot, t)|=\infty \quad \text { or } \quad \lim _{t \rightarrow T^{-}}|v(\cdot, t)|=\infty \quad \text { for all } x \in \Omega
$$

Secondly, we should point out that in the case of $m=n=0$, the system (1.1) becomes a semilinear system. To our knowledge, there do not seem to be any results in the literature on blow-up problems of these types. But other related works of the semilinear case have been deeply investigated by many authors, e.g., see $[11,12]$, and the authors of this paper in [13] studied the system

$$
u_{t}=\Delta u+a(x) u^{p_{1}}(x, t) v^{q_{1}}(0, t), \quad v_{t}=\Delta v+b(x) v^{p_{2}}(x, t) u^{q_{2}}(0, t),
$$

where the simultaneous and non-simultaneous blow-up criteria were obtained by using the fundamental solution of the heat equation. On the other hand, there are many known 
results concerning the global solutions and blow-up problems for the parabolic system with local nonlinearities, localized nonlinearities and nonlinear boundary conditions, see [14-17] and references therein. In particular, Ling and Wang in [18] considered the following degenerate parabolic system:

$$
u_{t}=\Delta u^{m}+v^{p_{1}}\|u\|_{\alpha}^{p_{2}}, \quad v_{t}=\Delta v^{n}+u^{q_{1}}\|v\|_{\beta}^{q_{2}}
$$

in a bounded domain $\Omega$, with the help of the super- and sub-solution methods, the critical exponent of the system was determined. Motivated by the above works, under the following conditions:

$$
0<m<p_{1}<1, \quad 0<n<q_{1}<1, \quad p_{2} q_{2}>\left(1-p_{1}\right)\left(1-q_{1}\right),
$$

we consider a more general degenerate parabolic system (1.1) which includes the problems considered in $[7,8]$ and [17] as special cases. Employing the ideas in $[7,8]$, we describe the blow-up rate of the radially symmetric solutions to (1.1). Here we discuss the blow-up of radially symmetric solutions as well as derive their blow-up rate. Moreover, we get the accurate coefficient of the blow-up rate. For the related discussion on a radially symmetric solution, we refer the readers to [19] and references therein.

In this paper, we always assume that the initial data $\left(u_{0}, v_{0}\right) \in \mathbf{V}$ ( $\mathbf{V}$ is defined by (1.8)) and satisfies the following $(\mathrm{H} 1)-(\mathrm{H} 3)$ or $(\mathrm{H} 4)$ :

(H1) $u_{0}(x), v_{0}(x) \in C^{2+\alpha}(B) \cap C(\bar{B}), \alpha \in(0,1)$.

(H2) $u_{0}(x), v_{0}(x)>0$ in $B, u_{0}(x)=v_{0}(x)=0, \frac{\partial u_{0}}{\partial v}, \frac{\partial v_{0}}{\partial v}<0$ on $\partial B$.

(H3) $u_{0}(x), v_{0}(x)$ are radially symmetric, $u_{0}^{\prime}(r), v_{0}^{\prime}(r) \leq 0$ for $r \in(0, R), r=|x|$.

Denote the set of initial data, depending only on the radial variable in the spherical coordinate system of $\mathbb{R}^{N}$ :

$$
\mathbf{V}=\left\{\left(u_{0}(r), v_{0}(r)\right) \mid \Phi_{1}(r)>0, \Phi_{2}(r)>0\right\}
$$

where

$$
\begin{aligned}
& \Phi_{1}(r)=m\left(u_{0}^{\prime}(r)\right)^{2} u_{0}^{m-1}(r)+u_{0}^{m}(r)\left(u_{0}^{\prime \prime}(r)+\frac{N-1}{r} u_{0}^{\prime}(r)\right)+a u_{0}^{p_{1}}(r)\left\|v_{0}(r)\right\|_{B, \alpha_{1}}^{p_{2}}, \\
& \Phi_{2}(r)=n\left(v_{0}^{\prime}(r)\right)^{2} v_{0}^{n-1}(r)+v_{0}^{n}(r)\left(v_{0}^{\prime \prime}(r)+\frac{N-1}{r} v_{0}^{\prime}(r)\right)+b v_{0}^{q_{1}}(r)\left\|u_{0}(r)\right\|_{B, \alpha_{2}}^{q_{2}} .
\end{aligned}
$$

It is noted that the set $\mathbf{V}$ is not empty. For example, for the simplest case $N=R=1$ and $a=b=1$, for any constant exponents $m, n$ and $p_{i}, q_{i}, \alpha_{i}, i=1,2$, there exist positive constants $a_{1}, a_{2}$ such that $\left(u_{0}, v_{0}\right) \in \mathbf{V}$ with $u_{0}(r)=a_{1} / 2-a_{1} r^{2} / 2, v_{0}(r)=a_{2} / 2-a_{2} r^{2} / 2$, $r \in[0,1)$.

(H4) Let $\delta_{0}, k_{1}, k_{2}$ be positive constants (will be given in Section 3), and there exists a constant $\delta>\delta_{0}$ such that

$$
\left\{\begin{array}{l}
\Delta u_{20}+a\left(1+m-p_{1}\right)\left\|v_{20}\right\|_{B, \mu_{1}}^{\sigma_{1}}-\delta u_{20}^{k_{1}+1-r_{1}} \geq 0 \\
\Delta v_{20}+b\left(1+n-q_{1}\right)\left\|u_{20}\right\|_{B, \mu_{2}}^{\sigma_{2}}-\delta v_{20}^{k_{2}+1-r_{2}} \geq 0
\end{array}\right.
$$

here $u_{20}, v_{20}$ and $\sigma_{i}, \mu_{i}, r_{i}$ are defined by (2.9) and (2.6). 
Then, our main results read as follows in detail.

Theorem 1 Assume that $\left(u_{0}, v_{0}\right) \in \mathbf{V}$ and satisfies (H1)-(H3). If $\rho^{2}>\frac{1}{a\left(1+m-p_{1}\right)} \frac{1}{b\left(1+n-q_{1}\right)}$, then the positive solution $(u, v)$ of (1.1) blows up in finite time, where $\rho$ is defined by (2.12).

Theorem 2 Under the assumptions of Theorem 1, if $\Delta u_{0}, \Delta v_{0} \leq 0$ on $\bar{B}$ and $\left(u_{0}, v_{0}\right)$ satisfies (H4), then the following statements hold uniformly on any compact subset of $B$ :

$$
\lim _{t \rightarrow T} \frac{u^{1-p_{1}}(x, t)}{\left(1-p_{1}\right) \widetilde{G}_{1}(t)}=a, \quad \lim _{t \rightarrow T} \frac{v^{1-q_{1}}(x, t)}{\left(1-q_{1}\right) \widetilde{G}_{2}(t)}=b,
$$

where $\widetilde{G}_{1}(t)$ and $\widetilde{G}_{2}(t)$ are defined by (3.1).

Theorem 3 Under the assumptions of Theorem 2, if $\left(1-q_{1}\right)\left(1+m-p_{1}\right)<p_{2}\left(q_{2}-m\right)$ and $\left(1-p_{1}\right)\left(1+n-q_{1}\right)<q_{2}\left(p_{2}-n\right)$, then

$$
\begin{gathered}
\lim _{t \rightarrow T} u(x, t)(T-t)^{\frac{1}{k_{1}}} \\
=\frac{\left(1+m-p_{1}\right)^{\frac{1}{1-p_{1}}}|B|^{\theta_{1}}}{d^{\frac{1}{k_{1}}}}\left(\frac{\beta_{2}}{a\left(1+m-p_{1}\right)}\right)^{\frac{1-q_{1}}{d}}\left(\frac{\beta_{1}}{b\left(1+n-q_{1}\right)}\right)^{\frac{p_{2}}{d}}, \\
\lim _{t \rightarrow T} v(x, t)(T-t)^{\frac{1}{k_{2}}} \\
=\frac{\left(1+n-q_{1}\right)^{\frac{1}{1-q_{1}}}|B|^{\theta_{2}}}{d^{\frac{1}{k_{2}}}}\left(\frac{\beta_{1}}{b\left(1+n-q_{1}\right)}\right)^{\frac{1-p_{1}}{d}}\left(\frac{\beta_{2}}{a\left(1+m-p_{1}\right)}\right)^{\frac{q_{2}}{d}},
\end{gathered}
$$

uniformly on compact subsets of $B$, where

$$
\begin{aligned}
& \theta_{1}=-\left(\frac{\sigma_{1}}{\mu_{1}}+\frac{\sigma_{1}}{\beta_{2}}\left(\frac{\sigma_{2}}{\mu_{2}}-\frac{\sigma_{1}}{\mu_{1}}\right)\right) \frac{1}{k_{1}}, \\
& \theta_{1}=-\left(\frac{\sigma_{2}}{\mu_{2}}+\frac{\sigma_{2}}{\beta_{1}}\left(\frac{\sigma_{1}}{\mu_{1}}-\frac{\sigma_{2}}{\mu_{2}}\right)\right) \frac{1}{k_{2}} .
\end{aligned}
$$

This paper is organized as follows. The result pertaining to blow-up of a solution in finite time is presented in Section 2, while results regarding the blow-up rates are established in Section 3. Some discussions are given in Section 4.

\section{Proof of Theorem 1}

In this section, we will discuss the blow-up of the solution to (1.1) and prove Theorem 1. By a simple computation, we have

$$
\begin{aligned}
& \nabla \cdot\left(u^{m} \nabla u\right)=m u^{m-1}|\nabla u|^{2}+u^{m} \Delta u, \\
& \Delta u^{1+m-p_{1}}=\left(1+m-p_{1}\right)\left(m-p_{1}\right) u^{m-p_{1}-1}|\nabla u|^{2}+\left(1+m-p_{1}\right) u^{m-p_{1}} \Delta u .
\end{aligned}
$$

Since $1+m-p_{1}>0, m<p_{1}$, from (2.2), we can derive the inequality

$$
u^{m-p_{1}} \Delta u \geq \frac{1}{1+m-p_{1}} \Delta u^{1+m-p_{1}} .
$$


Moreover, by (1.1), (2.1) and (2.3), we have

$$
\begin{aligned}
\frac{1}{1-p_{1}} \frac{\partial u^{1-p_{1}}}{\partial t} & =u^{-p_{1}} u_{t}=u^{-p_{1}}\left(m u^{m-1}|\nabla u|^{2}+u^{m} \Delta u+a u^{p_{1}}\|v\|_{B, \alpha_{1}}^{p_{2}}\right) \\
& \geq \frac{1}{1+m-p_{1}} \Delta u^{1+m-p_{1}}+a\|v\|_{B, \alpha_{1}}^{p_{2}} .
\end{aligned}
$$

Thus,

$$
\frac{1+m-p_{1}}{1-p_{1}} \frac{\partial\left(u^{1+m-p_{1}}\right)^{\frac{1-p_{1}}{1+m-p_{1}}}}{\partial t} \geq \Delta u^{1+m-p_{1}}+a\left(1+m-p_{1}\right)\|v\|_{B, \alpha_{1}}^{p_{2}}
$$

Similarly,

$$
\frac{1+n-q_{1}}{1-q_{1}} \frac{\partial\left(v^{1+n-q_{1}}\right)^{\frac{1-q_{1}}{1+n-q_{1}}}}{\partial t} \geq \Delta v^{1+n-q_{1}}+b\left(1+n-q_{1}\right)\|u\|_{B, \alpha_{2}}^{q_{2}}
$$

Denote $u_{1}=u^{1+m-p_{1}}, v_{1}=v^{1+n-q_{1}}$ and

$$
\left\{\begin{array}{lll}
r_{1}=\frac{m}{1+m-p_{1}}, & \sigma_{1}=\frac{p_{2}}{1+n-q_{1}}, & \mu_{1}=\frac{\alpha_{1}}{1+n-q_{1}} \\
r_{2}=\frac{n}{1+n-q_{1}}, & \sigma_{2}=\frac{q_{2}}{1+m-p_{1}}, & \mu_{2}=\frac{\alpha_{2}}{1+m-p_{1}} .
\end{array}\right.
$$

Then $0<r_{1}, r_{2}<1, \sigma_{1}, \sigma_{2}, \mu_{1}, \mu_{2}>1$ and $u_{1}, v_{1}$ satisfy

$$
\left\{\begin{array}{l}
u_{1 t} \geq u_{1}^{r_{1}}\left(\Delta u_{1}+a\left(1+m-p_{1}\right)\left\|v_{1}\right\|_{B, \mu_{1}}^{\sigma_{1}}\right) \\
v_{1 t} \geq v_{1}^{r_{2}}\left(\Delta v_{1}+b\left(1+n-q_{1}\right)\left\|u_{1}\right\|_{B, \mu_{2}}^{\sigma_{2}}\right)
\end{array}\right.
$$

Consider now the following problem:

$$
\left\{\begin{array}{l}
u_{2 t}=u_{2}^{r_{1}}\left(\Delta u_{2}+a\left(1+m-p_{1}\right)\left\|v_{2}\right\|_{B, \mu_{1}}^{\sigma_{1}}\right), \quad(x, t) \in B \times(0, T), \\
v_{2 t}=v_{2}^{r_{2}}\left(\Delta v_{2}+a\left(1+n-q_{1}\right)\left\|u_{2}\right\|_{B, \mu_{2}}^{\sigma_{2}}\right), \quad(x, t) \in B \times(0, T), \\
u_{2}(x, t)=v_{2}(x, t)=0, \quad(x, t) \in \partial B \times(0, T), \\
u_{2}(x, 0)=u_{20}(x), \quad v_{2}(x, 0)=v_{20}(x), \quad x \in B,
\end{array}\right.
$$

where

$$
\left\{\begin{array}{lr}
\left\|v_{2}\right\|_{B, \mu_{1}}^{\sigma_{1}}=\|v\|_{B, \alpha_{1}}^{p_{2}}, & \left\|u_{2}\right\|_{B, \mu_{2}}^{\sigma_{2}}=\|u\|_{B, \alpha_{2}}^{q_{2}}, \\
u_{20}(x)=\left(u_{0}(x)\right)^{1+m-p_{1}}, & v_{20}(x)=\left(v_{0}(x)\right)^{1+n-q_{1}} .
\end{array}\right.
$$

Since $u_{0}(x), v_{0}(x)$ satisfy $(\mathrm{H} 1)-(\mathrm{H} 2)$, then $(2.8)$ has a unique classical solution $\left(u_{2}, v_{2}\right)$ (see [20]). In the meantime, by the comparison principle, we observe

$$
u_{2}(x, t) \leq u^{1+m-p_{1}}(x, t), \quad v_{2}(x, t) \leq v^{1+n-q_{1}}(x, t), \quad(x, t) \in B \times(0, T) .
$$


Let $G$ be a bounded domain of $\mathbb{R}^{N}$. Consider the problem

$$
\begin{cases}\omega_{t}=d \omega^{r_{0}}\left(\Delta \omega+a_{0} \int_{G} \omega \mathrm{d} x\right), & x \in G, t>0, \\ \omega(x, t)=c, & x \in \partial G, t>0, \\ \omega(x, 0)=c, & x \in G,\end{cases}
$$

where $0<r_{0}<1$ and $d, a_{0}, c>0$ are some constants. By the standard method (see [3]), we can show that (2.11) has a unique classical solution $\omega(x, t)$ and $\omega(x, t) \geq c$. Denote by $\varphi_{0}(x)$ the unique positive solution of the linear elliptic problem

$$
-\Delta \varphi_{0}(x)=1, \quad x \in G ; \quad \varphi_{0}(x)=0, \quad x \in \partial G .
$$

Set $\rho_{0}=\int_{G} \varphi_{0}(x) \mathrm{d} x$, then we have:

Lemma 1 If $\rho_{0}>1 / a_{0}$, then the positive solution $\omega(x, t)$ of $(2.11)$ blows up in finite time.

Proof Set $H(t)=\int_{G} \omega^{1-r_{0}} \varphi_{0} \mathrm{~d} x$, then

$$
\begin{aligned}
\frac{1}{1-r_{0}} H^{\prime}(t) & =d\left(\int_{G} \Delta \omega \varphi_{0} \mathrm{~d} x+a_{0} \int_{G} \omega \mathrm{d} x \int_{G} \varphi_{0} \mathrm{~d} x\right) \\
& \geq d\left(a_{0} \rho_{0}-1\right) \int_{G} \omega \mathrm{d} x \geq d\left(a_{0} \rho_{0}-1\right)\left(\int_{G} \omega \varphi_{0} \mathrm{~d} x\right) / M,
\end{aligned}
$$

where $M=\max _{x \in \bar{G}} \varphi_{0}(x)$. Let $z=\omega^{1-r_{0}}$, then

$$
\int_{G} z_{t}(x, t) \varphi_{0}(x) \mathrm{d} x \geq d\left(1-r_{0}\right)\left(a_{0} \rho_{0}-1\right)\left(\int_{G} z^{1 /\left(1-r_{0}\right)} \varphi_{0} \mathrm{~d} x\right) / M .
$$

Since $1 /\left(1-r_{0}\right)>1$, from Jensen's inequality, it follows that

$$
\int_{G} z_{t}(x, t) \varphi_{0}(x) \mathrm{d} x \geq d\left(1-r_{0}\right)\left(a_{0} \rho_{0}-1\right)\left(\rho_{0}\right)^{-r_{0} /\left(1-r_{0}\right)}\left(\int_{G} z \varphi_{0} \mathrm{~d} x\right)^{1 /\left(1-r_{0}\right)} / M .
$$

That is $H^{\prime}(t) \geq C_{0} H^{1 /\left(1-r_{0}\right)}(t)$. In view of $H(0)>0$, it follows that there exists $T<\infty$ such that $\lim _{t \rightarrow T} H(t)=+\infty$, and hence $\omega(x, t)$ blows up in finite time.

Let $\varphi(x)$ be the unique positive solution of the following linear elliptic problem:

$$
-\Delta \varphi(x)=1, \quad x \in B, \quad \varphi(x)=0, \quad x \in \partial B
$$

and

$$
\rho=\min \left\{\rho_{01}=\|\varphi\|_{B, \mu_{1}}^{\sigma_{1}}, \rho_{02}=\|\varphi\|_{B, \mu_{2}}^{\sigma_{2}}\right\}
$$

Lemma 2 If $\rho^{2}>\frac{1}{a\left(1+m-p_{1}\right)} \frac{1}{b\left(1+n-q_{1}\right)}$, then for the solution $\left(u_{2}, v_{2}\right)$ of $(2.8)$, there exists a sufficiently small constant $\varepsilon>0$ such that

$$
\varepsilon \varphi(x) \leq u_{2}(x, t), \quad \varepsilon \varphi(x) \leq v_{2}(x, t)
$$

for all $(x, t) \in \bar{B} \times[0, T)$. 
Proof From (H1) and (H2) we see that there exists a sufficiently small constant $\varepsilon>0$ such that

$$
\varepsilon \varphi(x) \leq u_{20}(x), \quad \varepsilon \varphi(x) \leq v_{20}(x), \quad x \in \bar{B}
$$

and

$$
a\left(1+m-p_{1}\right) \rho>\varepsilon^{1-\sigma_{1}} \geq \varepsilon^{\sigma_{2}-1}>\left(b\left(1+n-q_{1}\right) \rho\right)^{-1} .
$$

Let $s_{1}(x, t)=\varepsilon \varphi(x), s_{2}(x, t)=\varepsilon \varphi(x)$, then we have by $(2.14)$

$$
\left\{\begin{array}{l}
s_{1 t}-s_{1}^{r_{1}}\left(\Delta s_{1}+a\left(1+m-p_{1}\right)\left\|s_{2}\right\|_{B, \mu_{1}}^{\sigma_{1}}\right) \leq s_{1}^{r_{1}}\left(\varepsilon-a\left(1+m-p_{1}\right) \varepsilon^{\sigma_{1}} \rho\right) \leq 0, \\
s_{2 t}-s_{2}^{r_{2}}\left(\Delta s_{2}+b\left(1+n-q_{1}\right)\left\|s_{1}\right\|_{B, \mu_{2}}^{\sigma_{2}}\right) \leq 0, \quad x \in B, 0<t<T, \\
s_{1}(x, t)=s_{2}(x, t)=0, \quad x \in \partial B, 0<t<T .
\end{array}\right.
$$

Thus it follows from (2.13) and (2.15) that $\left(s_{1}, s_{2}\right)$ is a sub-solution of (2.8). Hence, $(\varepsilon \varphi, \varepsilon \varphi) \leq\left(u_{2}, v_{2}\right)$ by the comparison principle.

Lemma 3 The solution $\left(u_{2}, v_{2}\right)$ of (2.8) blows up in finite time if $\rho^{2}>\frac{1}{a\left(1+m-p_{1}\right)} \frac{1}{b\left(1+n-q_{1}\right)}$ and $u_{0}, v_{0}$ satisfy $(H 1)-(H 3)$.

Proof In view of $\rho^{2}>\frac{1}{a\left(1+m-p_{1}\right)} \frac{1}{b\left(1+n-q_{1}\right)}$, we can choose a smooth sub-ball $B_{1} \subset \subset B$ such that

$$
\rho_{1}^{2}>\frac{1}{a\left(1+m-p_{1}\right)} \frac{1}{b\left(1+n-q_{1}\right)},
$$

where $\rho_{1}=\min \left\{\rho_{11}=\left\|\varphi_{1}\right\|_{B_{1}, \mu_{1}}^{\sigma_{1}}, \rho_{12}=\left\|\varphi_{1}\right\|_{B_{1}, \mu_{2}}^{\sigma_{2}}\right\}$ and $\varphi_{1}(x)>0$ satisfies

$$
-\Delta \varphi_{1}(x)=1, \quad x \in B_{1} ; \quad \varphi_{1}(x)=0, \quad x \in \partial B_{1} .
$$

On the other hand, there exists a sufficiently small $\varepsilon_{0}>0$ such that

$$
\int_{B_{1}} \varphi_{1}(x) \mathrm{d} x \geq \varepsilon_{0}\left\|\varphi_{1}\right\|_{B_{1}, \mu_{1}}^{\sigma_{1}}, \quad \int_{B_{1}} \varphi_{1}(x) \mathrm{d} x \geq \varepsilon_{0}\left\|\varphi_{1}\right\|_{B_{1}, \mu_{2}}^{\sigma_{2}} .
$$

Let $\eta=\varepsilon \min _{\bar{B}_{1}} \varphi$, here $\varepsilon$ is determined by Lemma 2 . Then $\eta>0$ and

$$
u_{2}(x, t) \geq \eta, \quad v_{2}(x, t) \geq \eta, \quad(x, t) \in \bar{B}_{1} \times(0, T)
$$

by Lemma 2 . Therefore, $\left(u_{2}, v_{2}\right)$ in $B_{1} \times(0, T)$ satisfies

$$
\left\{\begin{array}{l}
u_{2 t}=u_{2}^{r_{1}}\left(\Delta u_{2}+a\left(1+m-p_{1}\right)\left\|v_{2}\right\|_{B, \mu_{1}}^{\sigma_{1}}\right) \\
\quad \geq u_{2}^{r_{1}}\left(\Delta u_{2}+a\left(1+m-p_{1}\right)\left\|v_{2}\right\|_{B_{1}, \mu_{1}}^{\sigma_{1}}\right), \\
v_{2 t}=v_{2}^{r_{2}}\left(\Delta v_{2}+b\left(1+n-q_{1}\right)\left\|u_{2}\right\|_{B, \mu_{2}}^{\sigma_{2}}\right) \geq v_{2}^{r_{2}}\left(\Delta v_{2}+b\left(1+n-q_{1}\right)\left\|u_{2}\right\|_{B_{1}, \mu_{2}}^{\sigma_{2}}\right), \\
u_{2}(x, t) \geq \eta, \quad v_{2}(x, t) \geq \eta, \quad(x, t) \in \partial B_{1} \times(0, T), \\
u_{2}(x, 0)=u_{20}(x) \geq \eta, \quad v_{2}(x, 0)=v_{20}(x) \geq \eta, \quad x \in B_{1} .
\end{array}\right.
$$


Now, consider the following system:

$$
\begin{cases}u_{3 t}=u_{3}^{r_{1}}\left(\Delta u_{3}+a\left(1+m-p_{1}\right)\left\|v_{3}\right\|_{B_{1}, \mu_{1}}^{\sigma_{1}}\right), & x \in B_{1}, t>0, \\ v_{3 t}=v_{3}^{r_{2}}\left(\Delta v_{3}+b\left(1+n-q_{1}\right)\left\|u_{3}\right\|_{B_{1}, \mu_{2}}^{\sigma_{2}}\right), & x \in B_{1}, t>0, \\ u_{3}(x, t)=v_{3}(x, t)=\eta, & x \in \partial B_{1}, t>0, \\ u_{3}(x, 0)=v_{3}(x, 0)=\eta, & x \in B_{1} .\end{cases}
$$

Similarly, we can show that there exists a nonnegative classical solution $\left(u_{3}, v_{3}\right)$ of $(2.18)$ for $(x, t) \in B_{1} \times\left(0, T^{\prime}\right)$, where $T^{\prime}$ denotes the maximal existence time. The standard comparison principle for a parabolic system implies that $T^{\prime} \geq T$ and

$$
u_{2}(x, t) \geq u_{3}(x, t), \quad v_{2}(x, t) \geq v_{3}(x, t), \quad(x, t) \in \bar{B}_{1} \times(0, T) .
$$

Therefore, it suffices to show that $\left(u_{3}, v_{3}\right)$ blows up in finite time, because if so, its upper bound $\left(u_{2}, v_{2}\right)$ does exist up to a finite time $T$.

Since the initial data $(\eta, \eta)$ is a sub-solution of (2.18), the standard super-solution and sub-solution methods assert that $u_{3 t} \geq 0, v_{3 t} \geq 0$, which implies that

$$
\Delta u_{3}+a\left(1+m-p_{1}\right)\left\|v_{3}\right\|_{B_{1}, \mu_{1}}^{\sigma_{1}} \geq 0, \quad \Delta v_{3}+b\left(1+n-q_{1}\right)\left\|u_{3}\right\|_{B_{1}, \mu_{2}}^{\sigma_{2}} \geq 0
$$

Hence $u_{3}, v_{3} \geq \eta$ for $(x, t) \in \bar{B}_{1} \times\left[0, T^{\prime}\right)$. Thus, $\left(u_{3}, v_{3}\right)$ satisfies

$$
\left\{\begin{array}{l}
u_{3 t} \geq \eta^{r_{1}-r} u_{3}^{r}\left(\Delta u_{3}+a\left(1+m-p_{1}\right)\left\|v_{3}\right\|_{B_{1}, \mu_{1}}^{\sigma_{1}}\right), \quad(x, t) \in B_{1} \times\left(0, T^{\prime}\right) \\
v_{3 t} \geq \eta^{r_{2}-r} v_{3}^{r}\left(\Delta v_{3}+b\left(1+n-q_{1}\right)\left\|u_{3}\right\|_{B_{1}, \mu_{2}}^{\sigma_{2}}\right), \quad(x, t) \in B_{1} \times\left(0, T^{\prime}\right)
\end{array}\right.
$$

with the corresponding initial and boundary conditions and $0<r<\min \left\{r_{1}, r_{2}\right\}$.

Since $\rho_{1}^{2}>\frac{1}{a\left(1+m-p_{1}\right)} \frac{1}{b\left(1+n-q_{1}\right)}$, there exist positive constants $l_{1}, l_{2}$ with $l_{1}, l_{2}>1$, and $l$ such that

$$
\left\{\begin{array}{l}
a\left(1+m-p_{1}\right) \rho_{1}>\frac{l_{1}}{l_{2}}>\frac{1}{b\left(1+n-q_{1}\right) \rho_{1}}, \\
\rho_{1}>\frac{1}{l}>\frac{l_{1}\left|B_{1}\right|^{\frac{\mu_{1}-1}{\mu_{1}}}}{\varepsilon_{0} a\left(1+m-p_{1}\right) l_{2}^{\sigma_{1}}}, \quad \rho_{1}>\frac{1}{l}>\frac{l_{2}\left|B_{1}\right|^{\frac{\mu_{2}-1}{\mu_{2}}}}{\varepsilon_{0} b\left(1+n-q_{1}\right) l_{1}^{\sigma_{2}}} .
\end{array}\right.
$$

Let

$$
\omega_{1}(x, t)=l_{1} \omega(x, t), \quad \omega_{2}(x, t)=l_{2} \omega(x, t)
$$

where $\omega(x, t)$ is a unique positive solution of (2.11) with

$$
d=\min \left\{\eta^{r_{1}-r}, \eta^{r_{2}-r}\right\}, \quad r_{0}=r, \quad a_{0}=\frac{l}{\varepsilon_{0}}, \quad c=\min \left\{\frac{1}{l_{1}}, \frac{1}{l_{2}}\right\} \eta, \quad G=B_{1} \text {. }
$$

From (2.21) and Lemma 1, we know that $\omega(x, t)$ blows up in finite time $T_{0}<\infty$. Moreover, $\omega_{t} \geq 0$, that is, $\Delta \omega+a_{0} \int_{B_{1}} \omega \mathrm{d} x \geq 0$, since the initial data is a sub-solution of (2.11). In addition, from $\sigma_{1}, \sigma_{2}>1$ and Hölder's inequality, we have

$$
\left\{\begin{array}{l}
\int_{B_{1}} \omega \mathrm{d} x \leq\left|B_{1}\right|^{\frac{\mu_{1}-1}{\mu_{1}}}\left(\int_{B_{1}} \omega^{\mu_{1}} \mathrm{~d} x\right)^{\frac{1}{\mu_{1}}} \leq\left|B_{1}\right|^{\frac{\mu_{1}-1}{\mu_{1}}}\|\omega\|_{B_{1}, \mu_{1}}^{\sigma_{1}} \\
\int_{B_{1}} \omega \mathrm{d} x \leq\left|B_{1}\right|^{\frac{\mu_{2}-1}{\mu_{2}}}\left(\int_{B_{1}} \omega^{\mu_{2}} \mathrm{~d} x\right)^{\frac{1}{\mu_{2}}} \leq\left|B_{1}\right|^{\frac{\mu_{2}-1}{\mu_{2}}}\|\omega\|_{B_{1}, \mu_{2}}^{\sigma_{2}} .
\end{array}\right.
$$


Thus, a series of computations yields

$$
\left\{\begin{array}{l}
\omega_{1 t}-\eta^{r_{1}-r} \omega_{1}^{r}\left(\Delta \omega_{1}+a\left(1+m-p_{1}\right)\left\|\omega_{2}\right\|_{B_{1}, \mu_{1}}^{\sigma_{1}}\right) \\
\quad=l_{1} d \omega^{r}\left(\Delta \omega+\frac{l}{\varepsilon_{0}} \int_{B_{1}} \omega \mathrm{d} x\right)-l_{1} \eta^{r_{1}-r}\left(l_{1} \omega\right)^{r}\left(\Delta \omega+\frac{a\left(1+m-p_{1}\right) l_{2}^{\sigma_{1}}}{l_{1}}\|\omega\|_{B_{1}, \mu_{1}}^{\sigma_{1}}\right) \\
\quad \leq l_{1} d \omega^{r}\left(\Delta \omega+\frac{l}{\varepsilon_{0}}\left|B_{1}\right|^{\frac{\mu_{1}-1}{\mu_{1}}}\|\omega\|_{B_{1}, \mu_{1}}^{\sigma_{1}}\right) \\
\quad-l_{1} \eta^{r_{1}-r}\left(l_{1} \omega\right)^{r}\left(\Delta \omega+\frac{a\left(1+m-p_{1}\right) l_{2}^{\sigma_{1}}}{l_{1}}\|\omega\|_{B_{1}, \mu_{1}}^{\sigma_{1}}\right) \leq 0, \\
\omega_{2 t}-\eta^{r_{2}-r} \omega_{2}^{r}\left(\Delta \omega_{2}+b\left(1+n-q_{1}\right)\left\|\omega_{1}\right\|_{B_{1}, \mu_{2}}^{\sigma_{2}}\right) \leq 0, \quad x \in B_{1}, 0<t<T_{0}, \\
\omega_{1}(x, t)=l_{1} c \leq \eta, \quad \omega_{2}(x, t)=l_{2} c \leq \eta, \quad x \in \partial B_{1}, 0 \leq t<T_{0}, \\
\omega_{1}(x, 0)=l_{1} c \leq \eta, \quad \omega_{2}(x, 0)=l_{2} c \leq \eta, \quad x \in B_{1} .
\end{array}\right.
$$

It follows from (2.20), (2.23) and the comparison principle that $\left(\omega_{1}, \omega_{2}\right) \leq\left(u_{3}, v_{3}\right)$. Hence $\left(u_{3}, v_{3}\right)$ blows up in finite time, and so does the solution $\left(u_{2}, v_{2}\right)$ of (2.8) from (2.19). The proof now is completed.

Considering Lemma 3 and (2.10), we directly obtain the results of Theorem 1.

\section{Proofs of Theorems 2 and 3}

In this section, we assume that the solution $(u, v)$ of (1.1) blows up in finite time $T$ and will prove Theorems 2 and 3. We use $c$ or $C$ to denote the generic constant depending only on the structural data of the problem, and it may be different even in the same formula.

For the problem (1.1), denote

$$
\begin{aligned}
& \tilde{g}_{1}(t)=\|v\|_{B, \alpha_{1}}^{p_{2}}, \quad \tilde{g}_{2}(t)=\|u\|_{B, \alpha_{2}}^{q_{2}}, \\
& \widetilde{G}_{1}(t)=\int_{0}^{t} \tilde{g}_{1}(s) \mathrm{d} s, \quad \widetilde{G}_{2}(t)=\int_{0}^{t} \tilde{g}_{2}(s) \mathrm{d} s .
\end{aligned}
$$

Then we have

Lemma 4 Suppose that $u_{0}, v_{0}$ satisfy (H1)-(H3), then we have

$$
\lim _{t \rightarrow T} \widetilde{G}_{i}(t)=\lim _{t \rightarrow T} \sup \tilde{g}_{i}(t)=\infty, \quad i=1,2
$$

Proof According to the hypotheses, we know that $u(0, t) \geq u(x, t), v(0, t) \geq v(x, t),(x, t) \in$ $B \times(0, T)$. Let

$$
\widetilde{U}(t)=\max _{x \in \bar{B}} u(x, t)=u(0, t), \quad \widetilde{V}(t)=\max _{x \in \bar{B}} v(x, t)=v(0, t)
$$

Then, $\widetilde{U}(t), \widetilde{V}(t)$ are Lipschitz continuous (see [21]) and $\nabla \widetilde{U}=\nabla u(0, t)=0, \nabla \widetilde{V}=$ $\nabla v(0, t)=0$. Since $\left(u_{0}, v_{0}\right)$ is radially symmetric and non-increasing in $r=|x|,(u, v)$ is also a radially symmetric and non-increasing function, i.e., $u_{r}(r, t), v_{r}(r, t) \leq 0$ with $r=|x|$. Thus, $u(x, t)$ and $v(x, t)$ always reach their maxima at $x=0$, which means that $\Delta u(0, t), \Delta v(0, t) \leq 0$ for any $0<t<T$, i.e., $\Delta \widetilde{U}, \Delta \widetilde{V} \leq 0$ for any $0<t<T$. Therefore, it follows from (2.1) and (1.1) that

$$
\widetilde{U}^{\prime}(t) \leq a \widetilde{U}^{p_{1}}(t) \tilde{g}_{1}(t), \quad \widetilde{V}^{\prime}(t) \leq b \widetilde{V}^{q_{1}}(t) \tilde{g}_{2}(t) .
$$


Integrating (3.3) over $(0, t)$, we obtain

$$
\left\{\begin{aligned}
\frac{1}{1-p_{1}} \widetilde{U}^{1-p_{1}}(t) \leq a \widetilde{G}_{1}(t)+\frac{1}{1-p_{1}} \widetilde{U}^{1-p_{1}}(0), \\
\frac{1}{1-q_{1}} \widetilde{V}^{1-q_{1}}(t) \leq b \widetilde{G}_{2}(t)+\frac{1}{1-q_{1}} \widetilde{V}^{1-q_{1}}(0) .
\end{aligned}\right.
$$

From $\lim _{t \rightarrow T} \tilde{U}(t)=\lim _{t \rightarrow T} \tilde{V}(t)=\infty$ and $0<\tilde{U}(0), \widetilde{V}(0)<\infty$, we get

$$
\lim _{t \rightarrow T} \widetilde{G}_{i}(t)=\lim _{t \rightarrow T} \sup \tilde{g}_{i}(t)=\infty, \quad i=1,2
$$

Next, we first give some auxiliary lemmas about the solutions of (2.8), which will be used in the proofs of theorems. Similar to (3.2), we let

$$
U(t)=\max _{x \in \bar{B}} u_{2}(x, t), \quad V(t)=\max _{x \in \bar{B}} v_{2}(x, t)
$$

By (2.8), we see that $U(t)$ and $V(t)$ satisfy

$$
\begin{aligned}
& U_{t} \leq a\left(1+m-p_{1}\right)|B|^{\frac{\sigma_{1}}{\mu_{1}}} U^{r_{1}} V^{\sigma_{1}}, \\
& V_{t} \leq b\left(1+n-q_{1}\right)|B|^{\frac{\sigma_{2}}{\mu_{2}}} V^{r_{2}} U^{\sigma_{2}}, \quad \text { a.e. } t \in(0, T) .
\end{aligned}
$$

Let $\beta_{1}=1-r_{1}+\sigma_{2}, \beta_{2}=1-r_{2}+\sigma_{1}$, then $\beta_{1}, \beta_{2}>0$. By Young's inequality, we have

$$
\begin{aligned}
\left(U^{\beta_{1}}+V^{\beta_{2}}\right)_{t} & \leq\left(\beta_{1} a\left(1+m-p_{1}\right)|B|^{\frac{\sigma_{1}}{\mu_{1}}}+\beta_{2} b\left(1+n-q_{1}\right)|B|^{\frac{\sigma_{2}}{\mu_{2}}}\right) U^{\beta_{1} \frac{\sigma_{2}}{\beta_{1}}} V^{\beta_{2} \frac{\sigma_{1}}{\beta_{2}}} \\
& \leq C\left(U^{\beta_{1}}+V^{\beta_{2}}\right)^{\frac{\sigma_{2}}{\beta_{1}}+\frac{\sigma_{1}}{\beta_{2}}} .
\end{aligned}
$$

Integrating the above inequality over $(t, T)$, we obtain

$$
U^{\beta_{1}}+V^{\beta_{2}} \geq C(T-t)^{-\frac{\beta_{1} \beta_{2}}{d}}
$$

where $d=\sigma_{1} \sigma_{2}-\left(1-r_{1}\right)\left(1-r_{2}\right)>0$ by $(1.7)$.

Lemma 5 Suppose that $u_{0}, v_{0}$ satisfy (H1)-(H3) and the solution $\left(u_{2}, v_{2}\right)$ of (2.8) blows up in finite time T. Then, we have

$$
\lim _{t \rightarrow T} \sup g_{i}(t)=\lim _{t \rightarrow T} G_{i}(t)=\infty, \quad i=1,2,
$$

where

$$
\begin{aligned}
& g_{1}(t)=\left\|v_{2}\right\|_{B, \mu_{1}}^{\sigma_{1}}, \quad g_{2}(t)=\left\|u_{2}\right\|_{B, \mu_{2}}^{\sigma_{2}}, \\
& G_{1}(t)=\int_{0}^{t} g_{1}(s) \mathrm{d} s, \quad G_{2}(t)=\int_{0}^{t} g_{2}(s) \mathrm{d} s .
\end{aligned}
$$

Proof Let $U(t), V(t)$ be as (3.5), then from (2.8), we have

$$
\begin{aligned}
& U^{\prime}(t) \leq a\left(1+m-p_{1}\right) U^{r_{1}}(t) g_{1}(t), \\
& V^{\prime}(t) \leq b\left(1+n-q_{1}\right) V^{r_{2}}(t) g_{2}(t), \quad \text { a.e. } t \in[0, T) .
\end{aligned}
$$


Integrating (3.9) over $(0, t)$, we obtain

$$
\left\{\begin{array}{l}
\frac{1}{1-r_{1}} U^{1-r_{1}}(t) \leq a\left(1+m-p_{1}\right) G_{1}(t)+\frac{1}{1-r_{1}} U^{1-r_{1}}(0) \\
\frac{1}{1-r_{2}} V^{1-r_{2}}(t) \leq b\left(1+n-q_{1}\right) G_{2}(t)+\frac{1}{1-r_{2}} V^{1-r_{2}}(0)
\end{array}\right.
$$

Similar to the proofs of $\widetilde{G}_{i}(t)$ and $\tilde{g}_{i}(t)$, we have

$$
\lim _{t \rightarrow T} G_{i}(t)=\lim _{t \rightarrow T} \sup g_{i}(t)=\infty, \quad i=1,2 .
$$

Lemma 6 Suppose that $u_{0}, v_{0}$ satisfy (H1)-(H4). Then, we have

$$
u_{2 t}-\delta u_{2}^{k_{1}+1} \geq 0, \quad v_{2 t}-\delta v_{2}^{k_{2}+1} \geq 0, \quad(x, t) \in B \times(0, T),
$$

here $k_{1}=d / \beta_{2}, k_{2}=d / \beta_{1}$.

Proof Set $J_{1}(x, t)=u_{2 t}-\delta u_{2}^{k_{1}+1}, J_{2}(x, t)=u_{2 t}-\delta u_{2}^{k_{2}+1}$. Then,

$$
\lim _{x \rightarrow \partial B} J_{1}(x, t) \geq 0, \quad \lim _{x \rightarrow \partial B} J_{2}(x, t) \geq 0 ; \quad J_{1}(x, 0) \geq 0, J_{2}(x, 0) \geq 0, x \in B .
$$

A series of computations yields $J_{1 t}=u_{2 t t}-\delta\left(k_{1}+1\right) u_{2}^{k_{1}} u_{2 t}$ and

$$
\begin{aligned}
u_{2 t t}= & r_{1} u_{2}^{-1}\left(J_{1}^{2}+2 \delta u_{2}^{k_{1}+1} J_{1}+\delta^{2} u_{2}^{2 k_{1}+2}\right)+u_{2}^{r_{1}} \Delta J_{1}+\delta\left(k_{1}+1\right) k_{1} u_{2}^{k_{1}-1+r_{1}}\left|\nabla u_{2}\right|^{2} \\
& +\delta\left(k_{1}+1\right) u_{2}^{k_{1}+r_{1}} \Delta u_{2}+a\left(1+m-p_{1}\right) \sigma_{1} u_{2}^{r_{1}}\left\|v_{2}\right\|_{\mu_{1}}^{\sigma_{1}-\mu_{1}} \int_{B} v_{2}^{\mu_{1}-1}\left(J_{2}+\delta v_{2}^{k_{2}+1}\right) \mathrm{d} x \\
= & u_{2}^{r_{1}} \Delta J_{1}+\left(2 r_{1} \delta u_{2}^{k_{1}}+\delta\left(k_{1}+1\right) u_{2}^{k_{1}}\right) J_{1}+r_{1} u_{2}^{-1} J_{1}^{2}+\delta\left(k_{1}+1\right) k_{1} u_{2}^{k_{1}-1+r_{1}}\left|\nabla u_{2}\right|^{2} \\
& +\left(r_{1} \delta^{2}+\left(k_{1}+1\right) \delta^{2}\right) u_{2}^{2 k_{1}+1}-\left(k_{1}+1\right) a\left(1+m-p_{1}\right) \delta u_{2}^{k_{1}+r_{1}}\left\|v_{2}\right\|_{\mu_{1}}^{\sigma_{1}} \\
& +a\left(1+m-p_{1}\right) \sigma_{1} u_{2}^{r_{1}}\left\|v_{1}\right\|_{\mu_{1}}^{\sigma_{1}-\mu_{1}} \int_{B} v_{2}^{\mu_{1}-1} J_{2} \mathrm{~d} x \\
& +a\left(1+m-p_{1}\right) \sigma_{1} \delta u_{2}^{r_{1}}\left\|v_{2}\right\|_{\mu_{1}}^{\sigma_{1}-\mu_{1}}\left\|v_{2}\right\|_{\mu_{1}+k_{2}}^{\mu_{1}+k_{2}} .
\end{aligned}
$$

From the condition (1.7), it is easy to calculate that $k_{1}+1>r_{1}$. Then, it entails

$$
\begin{aligned}
J_{1 t}- & u_{2}^{r_{1}} \Delta J_{1}-2 r_{1} \delta u_{2}^{k_{1}} J_{1}-a\left(1+m-p_{1}\right) \sigma_{1} u_{2}^{r_{1}}\left\|v_{2}\right\|_{\mu_{1}}^{\sigma_{1}-\mu_{1}} \int_{B} v_{2}^{\mu_{1}-1} J_{2} \mathrm{~d} x \\
\geq & r_{1} \delta^{2} u_{2}^{2 k_{1}+1}+a\left(1+m-p_{1}\right) \sigma_{1} \delta u_{2}^{r_{1}}\left\|v_{2}\right\|_{\mu_{1}}^{\sigma_{1}-\mu_{1}}\left\|v_{2}\right\|_{\mu_{1}+k_{2}}^{\mu_{1}+k_{2}} \\
& \quad-\left(k_{1}+1\right) a\left(1+m-p_{1}\right) \delta u_{2}^{k_{1}+r_{1}}\left\|v_{2}\right\|_{\mu_{1}}^{\sigma_{1}} \\
= & a\left(1+m-p_{1}\right)\left(k_{1}+1\right) \delta u_{2}^{r_{1}}\left(\frac{r_{1} \delta u_{2}^{2 k_{1}+1-r_{1}}}{a\left(1+m-p_{1}\right)\left(k_{1}+1\right)}\right. \\
& \left.+\frac{\sigma_{1}}{k_{1}+1}\left\|v_{2}\right\|_{\mu_{1}}^{\sigma_{1}-\mu_{1}}\left\|v_{2}\right\|_{\mu_{1}+k_{2}}^{\mu_{1}+k_{2}}-u_{2}^{k_{1}}\left\|v_{2}\right\|_{\mu_{1}}^{\sigma_{1}}\right) .
\end{aligned}
$$


By the Hölder inequality, for any $0<\theta<1$, it follows that

$$
\left\|v_{2}\right\|_{\mu_{1}}^{\sigma_{1}}=\left\|v_{2}\right\|_{\mu_{1}}^{\left(\sigma_{1}-\mu_{1}\right) \theta}\left\|v_{2}\right\|_{\mu_{1}}^{\sigma_{1}-\left(\sigma_{1}-\mu_{1}\right) \theta} \leq\left\|v_{2}\right\|_{\mu_{1}}^{\left(\sigma_{1}-\mu_{1}\right) \theta}\left\|v_{2}\right\|_{\mu_{1}+k_{2}}^{\sigma_{1}-\left(\sigma_{1}-\mu_{1}\right) \theta}|B|^{\frac{k_{2}\left[\sigma_{1}-\left(\sigma_{1}-\mu_{1}\right) \theta\right]}{\mu_{1}\left(\mu_{1}+k_{2}\right)}} .
$$

Furthermore, by Young's inequality, for any $\varepsilon>0$ and $l_{1}, l_{2}>1$ satisfying $1 / l_{1}+1 / l_{2}=1$, the following inequality holds:

$$
\begin{aligned}
u_{2}^{k_{1}}\left\|v_{2}\right\|_{\mu_{1}}^{\sigma_{1}} & \leq u_{2}^{k_{1}}\left\|v_{2}\right\|_{\mu_{1}}^{\left(\sigma_{1}-\mu_{1}\right) \theta}\left\|v_{2}\right\|_{\mu_{1}+k_{2}}^{\sigma_{1}-\left(\sigma_{1}-\mu_{1}\right) \theta}|B|^{\frac{k_{2}\left[\sigma_{1}-\left(\sigma_{1}-\mu_{1}\right) \theta\right]}{\mu_{1}\left(\mu_{1}+k_{2}\right)}} \\
& \leq|B|^{\frac{k_{2}\left[\sigma_{1}-\left(\sigma_{1}-\mu_{1}\right) \theta\right]}{\mu_{1}\left(\mu_{1}+k_{2}\right)}}\left(\frac{\left(\varepsilon u_{2}^{k_{1}}\right)^{l_{1}}}{l_{1}}+\frac{1}{l_{2}}\left(\frac{1}{\varepsilon}\left\|v_{2}\right\|_{\mu_{1}}^{\left(\sigma_{1}-\mu_{1}\right) \theta}\left\|v_{2}\right\|_{\mu_{1}+k_{2}}^{\sigma_{1}-\left(\sigma_{1}-\mu_{1}\right) \theta}\right)^{l_{2}}\right) .
\end{aligned}
$$

Now, we take

$$
l_{1}=\frac{k_{2}+\sigma_{1}}{k_{2}}, \quad l_{2}=\frac{k_{2}+\sigma_{1}}{\sigma_{1}}, \quad \theta=\frac{\sigma_{1}}{k_{2}+\sigma_{1}}, \quad \varepsilon=\left(\frac{k_{1}+1}{k_{2}+\sigma_{1}}|B|^{\frac{k_{2} \sigma_{1}}{\mu_{1}\left(k_{2}+\sigma_{1}\right)}}\right)^{\frac{\sigma_{1}}{k_{2}+\sigma_{1}}}
$$

Therefore, by (3.12) and (3.13), it follows that

$$
\begin{aligned}
J_{1 t} & -u_{2}^{r_{1}} \Delta J_{1}-2 r_{1} \delta u_{2}^{k_{1}} J_{1}-a\left(1+m-p_{1}\right) \sigma_{1} u_{2}^{r_{1}}\left\|v_{2}\right\|_{\mu_{1}}^{\sigma_{1}-\mu_{1}} \int_{B} v_{2}^{\mu_{1}-1} J_{2} \mathrm{~d} x \\
& \geq r_{1} \delta\left(\delta-\delta_{1}\right) u_{2}^{2 k_{1}+2} \geq 0,
\end{aligned}
$$

where

$$
\delta_{1}=\frac{a\left(1+m-p_{1}\right) k_{2}}{r_{1}}|B|^{\frac{\sigma_{1}}{\mu_{1}}}\left(\frac{k_{1}+1}{k_{2}+\sigma_{1}}\right)^{\frac{\sigma_{1}}{k_{2}}+1} .
$$

We can determine a number $\delta_{2}$ in the similar way. Let $\delta_{0}=\max \left\{\delta_{1}, \delta_{2}\right\}$, similar to the above, one has

$$
J_{2 t}-v_{2}^{r_{2}} \Delta J_{2}-2 r_{2} \delta v_{2}^{k_{2}} J_{2}-b\left(1+n-q_{1}\right) \sigma_{2} v_{2}^{r_{2}}\left\|u_{2}\right\|_{\mu_{2}}^{\sigma_{2}-\mu_{2}} \int_{B} u_{2}^{\mu_{2}-1} J_{1} \mathrm{~d} x \geq 0 .
$$

By the comparison principle of Lemma 1 in [20], we have $J_{1}, J_{2} \geq 0$. This completes the proof.

Lemma 7 Suppose that $u_{0}, v_{0}$ satisfy (H1)-(H4), then there exist positive constants $c$ and C such that

$$
\left\{\begin{array}{l}
c \leq \max _{x \in \bar{B}} u_{2}(x, t)(T-t)^{1 / k_{1}} \leq C, \\
c \leq \max _{x \in \bar{B}} v_{2}(x, t)(T-t)^{1 / k_{2}} \leq C .
\end{array}\right.
$$

Proof It follows from (3.11) that

$$
U_{t} \geq \delta U^{k_{1}+1}, \quad V_{t} \geq \delta V^{k_{2}+1}, \quad t \in(0, T)
$$

Combining with (3.6), we can obtain

$$
U^{k_{1}+1-r_{1}} \leq \frac{a\left(1+m-p_{1}\right)}{\delta}|B|^{\frac{\sigma_{1}}{\mu_{1}}} V^{\sigma_{1}}, \quad V^{k_{2}+1-r_{2}} \leq \frac{b\left(1+n-q_{1}\right)}{\delta}|B|^{\frac{\sigma_{2}}{\mu_{2}}} U^{\sigma_{2}} .
$$


The direct computation yields $k_{1}+1-r_{1}=\sigma_{1} \beta_{1} / \beta_{2}, k_{2}+1-r_{1}=\sigma_{2} \beta_{2} / \beta_{1}$. It follows from (3.16) that

$$
U^{\beta_{1}} \leq\left(\frac{a\left(1+m-p_{1}\right)}{\delta}\right)^{\frac{\beta_{2}}{\sigma_{1}}}|B|^{\frac{\beta_{2}}{\mu_{1}}} V^{\beta_{2}}, \quad V^{\beta_{2}} \leq\left(\frac{b\left(1+n-q_{1}\right)}{\delta}\right)^{\frac{\beta_{1}}{\sigma_{2}}}|B|^{\frac{\beta_{1}}{\mu_{2}}} U^{\beta_{1}} .
$$

Therefore, combining (3.17) with (3.7) gives

$$
c \leq U(t)(T-t)^{1 / k_{1}}, \quad c \leq V(t)(T-t)^{1 / k_{2}} .
$$

Integrating (3.15) from $t$ to $T$, we end the proof.

Lemma 8 Suppose that $u_{0}, v_{0}$ satisfy (H1)-(H4) and $\Delta u_{0}, \Delta v_{0} \leq 0$, then

$$
\lim _{t \rightarrow T} \frac{u_{2}^{1-r_{1}}(x, t)}{\left(1-r_{1}\right) G_{1}(t)}=a\left(1+m-p_{1}\right), \quad \lim _{t \rightarrow T} \frac{v_{2}^{1-r_{2}}(x, t)}{\left(1-r_{2}\right) G_{2}(t)}=b\left(1+n-q_{1}\right)
$$

uniformly on compact subsets of $B$.

Proof Here we consider the first eigenvalue problem

$$
-\Delta \phi(x)=\lambda_{1} \phi(x), \quad x \in B ; \quad \phi(x)=0, \quad x \in \partial B .
$$

Normalize $\phi(x)$ as $\phi(x)>0$ in $B$ and $\int_{B} \phi(x) \mathrm{d} x=1$. Define

$$
z(x, t)=a\left(1+m-p_{1}\right) G_{1}(t)-\frac{1}{1-r_{1}} u_{2}^{1-r_{1}}(x, t), \quad \gamma(t)=\int_{B} z(y, t) \phi(y) \mathrm{d} y .
$$

A series of computations yields

$$
\begin{aligned}
\gamma^{\prime}(t) & =\int_{B}\left(a\left(1+m-p_{1}\right) g_{1}(t)-u_{2}^{-r_{1}} u_{2 t}\right) \phi(y) \mathrm{d} y \\
& =-\int_{B} \Delta u_{2}(y, t) \phi(y) \mathrm{d} y=\lambda_{1} \int_{B} u_{2}(y, t) \phi(y) \mathrm{d} y \\
& =\lambda_{1}\left(1-r_{1}\right)^{\frac{1}{1-r_{1}}} \int_{B}\left(a\left(1+m-p_{1}\right) G_{1}(t)-z(y, t)\right)^{\frac{1}{1-r_{1}}} \phi(y) \mathrm{d} y \\
& \leq \lambda_{1}\left(1-r_{1}\right)^{\frac{1}{1-r_{1}}} \int_{B}\left(a\left(1+m-p_{1}\right) G_{1}(t)+z^{-}(y, t)\right)^{\frac{1}{1-r_{1}}} \phi(y) \mathrm{d} y \\
& \leq C\left(G_{1}^{\frac{1}{1-r_{1}}}(t)+\int_{B}\left(z^{-}(y, t)\right)^{\frac{1}{1-r_{1}}} \phi(y) \mathrm{d} y\right),
\end{aligned}
$$

where $z^{-}=\max \{-z, 0\}$. By (3.10), we know that $\inf _{B} z(x, t) \geq-C$, which means $z^{-}(x, t) \leq C$. Then

$$
\gamma^{\prime}(t) \leq C G_{1}^{\frac{1}{1-r_{1}}}(t)+C
$$

Integrating (3.19) from 0 to $t$ yields

$$
\gamma(t) \leq C\left(1+\int_{0}^{t} G_{1}^{\frac{1}{1-r_{1}}}(s) \mathrm{d} s\right)
$$


That is

$$
\int_{B}|z(y, t)| \phi(y) \mathrm{d} y \leq C\left(1+\int_{0}^{t} G_{1}^{\frac{1}{1-r_{1}}}(s) \mathrm{d} s\right) .
$$

Denote $B_{\varrho}=\{y \in B: \varrho \leq|y|<R\}$. Since $-\Delta z \leq 0$, using Lemma 4.5 in [4], we obtain

$$
\sup _{B_{\varrho}} z(x, t) \leq \frac{C}{\varrho^{N+1}}\left(1+\int_{0}^{t} G_{1}^{\frac{1}{1-r_{1}}}(s) \mathrm{d} s\right)
$$

It follows from (3.21) and (3.10) that

$$
-\frac{C}{G_{1}(t)} \leq a\left(1+m-p_{1}\right)-\frac{u_{2}^{1-r_{1}}}{\left(1-r_{1}\right) G_{1}(t)} \leq \frac{C\left(1+\int_{0}^{t} G_{1}^{\frac{1}{1-r_{1}}}(s) \mathrm{d} s\right)}{G_{1}(t)}
$$

for any $x \in B_{\varrho}$. On the other hand, we know from (3.10), (3.14) and $\Delta u_{0}, \Delta v_{0} \leq 0$ that

$$
c \leq(T-t)^{\frac{\left(1-r_{1}\right)\left(1-r_{2}+\sigma_{1}\right)}{d}} G_{1}(t) \leq C .
$$

Therefore,

$$
\left\{\begin{array}{l}
c \leq(T-t)^{\frac{1-r_{2}+\sigma_{1}}{d}} G_{1}^{\frac{1}{1-r_{1}}}(t) \leq C, \\
c \leq(T-t)^{\frac{\left(1-r_{1}\right)\left(1-r_{2}+\sigma_{1}\right)}{d}+1} G_{1}^{\prime}(t) \leq C .
\end{array}\right.
$$

Noting that

$$
\frac{1-r_{2}+\sigma_{1}}{d}<\frac{\left(1-r_{1}\right)\left(1-r_{2}+\sigma_{1}\right)}{d}+1 \Longleftrightarrow 1-r_{2}<\sigma_{1}\left(\sigma_{2}-r_{1}\right)
$$

Then

$$
\lim _{t \rightarrow T} \frac{\int_{0}^{t} G_{1}^{\frac{1}{1-r_{1}}}(s) \mathrm{d} s}{G_{1}(t)}=\lim _{t \rightarrow T} \frac{G_{1}^{\frac{1}{1-r_{1}}}(t)}{G_{1}^{\prime}(t)}=0 .
$$

Thus

$$
\lim _{t \rightarrow T} \frac{u_{2}^{1-r_{1}}(x, t)}{\left(1-r_{1}\right) G_{1}(t)}=a\left(1+m-p_{1}\right) .
$$

Similarly,

$$
\lim _{t \rightarrow T} \frac{v_{2}^{1-r_{2}}(x, t)}{\left(1-r_{2}\right) G_{2}(t)}=b\left(1+n-q_{1}\right) .
$$

Proof of Theorem 2 According to $u_{2} \leq u^{1+m-p_{1}}$, it follows from (2.9), (3.1), (3.8) and (3.18) that

$$
\liminf _{t \rightarrow T} \frac{u^{1-p_{1}}(x, t)}{\left(1-p_{1}\right) \widetilde{G}_{1}(t)} \geq \lim _{t \rightarrow T} \frac{u_{2}^{1-r_{1}}(x, t)}{\left(1-r_{1}\right) G_{1}(t)} \frac{1}{1+m-p_{1}}=a .
$$


On the other hand, from (3.4), we estimate

$$
\lim _{t \rightarrow T} \sup \frac{\widetilde{U}^{1-p_{1}}(t)}{\left(1-p_{1}\right) \widetilde{G}_{1}(t)} \leq a .
$$

Combining (3.23) with (3.24), we obtain

$$
\lim _{t \rightarrow T} \frac{u^{1-p_{1}}(x, t)}{\left(1-p_{1}\right) \widetilde{G}_{1}(t)}=a
$$

Similarly,

$$
\lim _{t \rightarrow T} \frac{v^{1-q_{1}}(x, t)}{\left(1-q_{1}\right) \widetilde{G}_{2}(t)}=b .
$$

This completes the proof of the theorem.

Proof of Theorem 3 By Theorem 2, we have that, as $t \rightarrow T$,

$$
\left\{\begin{array}{l}
\widetilde{G}_{1}^{\prime}(t)=\|v\|_{B, \alpha_{1}}^{p_{2}} \sim|B|^{\frac{p_{2}}{\alpha_{1}}}\left(b\left(1-q_{1}\right)\right)^{\frac{p_{2}}{1-q_{1}}} \widetilde{G}_{2}^{\frac{p_{2}}{1-q_{1}}}(t) \\
\widetilde{G}_{2}^{\prime}(t)=\|u\|_{B, \alpha_{2}}^{q_{2}} \sim|B|^{\frac{q_{2}}{\alpha_{2}}}\left(a\left(1-p_{1}\right)\right)^{\frac{q_{2}}{1-p_{1}}} \widetilde{G}_{1}^{\frac{q_{2}}{1-p_{1}}}(t)
\end{array}\right.
$$

where the notation $u \sim v$ means that $\lim _{t \rightarrow T} u(t) / v(t)=1$. Hence, we obtain

$$
\frac{\mathrm{d} \widetilde{G}_{1}}{\mathrm{~d} \widetilde{G}_{2}} \sim|B|^{\frac{p_{2}}{\alpha_{1}}-\frac{q_{2}}{\alpha_{2}}}\left(a\left(1-p_{1}\right)\right)^{-\frac{q_{2}}{1-p_{1}}}\left(b\left(1-q_{1}\right)\right)^{\frac{p_{2}}{1-q_{1}}} \widetilde{G}_{1}^{-\frac{q_{2}}{1-p_{1}}} \widetilde{G}_{2}^{\frac{p_{2}}{1-q_{1}}} .
$$

A series of computations yields

$$
\left\{\begin{array}{l}
\widetilde{G}_{1}(t) \sim \frac{|B|^{\theta_{1}\left(1-p_{1}\right)} d^{-\frac{\beta_{2}\left(1-p_{1}\right)}{d}}}{a\left(1-p_{1}\right)\left(1+m-p_{1}\right)}\left(\frac{\beta_{2}}{a\left(1+m-p_{1}\right)}\right)^{\frac{\left(1-p_{1}\right)\left(1-q_{1}\right)}{d}}\left(\frac{\beta_{1}}{b\left(1+n-q_{1}\right)}\right)^{\frac{p_{2}\left(1-p_{1}\right)}{d}}(T-t)^{-\frac{\beta_{2}\left(1-p_{1}\right)}{d}}, \\
\widetilde{G}_{2}(t) \sim \frac{\mid B B_{2}\left(1-q_{1}\right) d^{-\frac{\beta_{1}\left(1-q_{1}\right)}{d}}}{b\left(1-q_{1}\right)\left(1+n-q_{1}\right)}\left(\frac{\beta_{1}}{b\left(1+n-q_{1}\right)}\right) \frac{\left(1-p_{1}\right)\left(1-q_{1}\right)}{d}\left(\frac{\beta_{2}}{a\left(1+m-p_{1}\right)}\right)^{\frac{q_{2}\left(1-q_{1}\right)}{d}}(T-t)^{-\frac{\beta_{1}\left(1-q_{1}\right)}{d}} .
\end{array}\right.
$$

Combining with Lemma 7, we obtain the results of Theorem 3 immediately.

\section{Discussions}

The results in this paper show the interactions among the multi-nonlinearities in the reaction-diffusion system (1.1). Roughly speaking, either large exponents $m, n$, large coupling exponents $p_{2}, q_{2}$ or large constants $a, b$ benefit from the occurrence of the finite blow-up. For example, to make a finite blow-up to the problem (1.1), for fixed $m, p_{1}, p_{2}$, $\alpha_{1}$ and $n, q_{1}, q_{2}, \alpha_{2}$, constants $a$ and $b$ should be properly large such that the following inequality

$$
\rho^{2}>\frac{1}{a\left(1+m-p_{1}\right)} \frac{1}{b\left(1+n-q_{1}\right)}
$$

holds. 


\section{Competing interests}

The authors declare that they have no competing interests.

\section{Authors' contributions}

The authors declare that the study was realized in collaboration with the same responsibility. All authors read and approved the final manuscript.

\section{Author details}

${ }^{1}$ College of Mathematics and Information Science, Yulin Normal University, Yulin, Guangxi 537000, P.R. China. ${ }^{2}$ College of Mathematics and Information Science, Jiangxi Normal University, Nanchang, 330022, P.R. China.

\section{Acknowledgements}

The authors are supported by National Natural Science Foundation of China and they would like to express their many thanks to the editor and reviewers for their constructive suggestions to improve the previous version of this paper. This work is supported by the NNSF of China (11071100).

\section{Received: 19 May 2012 Accepted: 29 August 2012 Published: 11 September 2012}

\section{References}

1. Escobedo, M, Herrero, M: Boundedness and blow-up for a semilinear reaction-diffusion system. J. Differ. Equ. 89(1), 176-202 (1991). doi:10.1016/0022-0396(91)90118-S

2. Galaktionov, V, Kurdyumov, S, Samarskii, A: A parabolic system of quasilinear equations I. Differ. Equ. 19, 1558-1574 (1984)

3. Souplet, P: Blow up in nonlocal reaction diffusion equations. SIAM J. Math. Anal. 29, 1301-1334 (1998)

4. Souplet, P: Uniform blow-up profiles and boundary behavior for diffusion equations with nonlocal nonlinear sources. J. Differ. Equ. 153, 374-406 (1999). doi:10.1006/jdeq.1998.3535

5. Pao, C: Nonexistence of global solutions for an integrodifferential system in reactor dynamics. SIAM J. Math. Anal. 11(3), 559-564 (1980)

6. Guo, JS, Su, HW: The blow-up behaviour of the solution of an integrodifferential equation. Differ. Integral Equ. 5(6), 1237-1245 (1992)

7. Li, F, Xie, CH: Global existence and blow-up for a nonlinear porous medium equation. Appl. Math. Lett. 16, 185-192 (2003). doi:10.1016/S0893-9659(03)80030-7

8. Liu, QL, Li, YX, Gao, HG: Uniform blow-up rate for a nonlocal degenerate parabolic equations. Nonlinear Anal. TMA 66, 881-889 (2007). doi:10.1016/j.na.2005.12.029

9. Deng, WB, Li, YX, Xie, CH: Existence and nonexistence of global solutions of some nonlocal degenerate parabolic equations. Appl. Math. Lett. 16, 803-808 (2003). doi:10.1016/S0893-9659(03)80118-0

10. Liu, QL, Li, YX, Gao, HG: Uniform blow-up rate for diffusion equations with nonlocal nonlinear source. Nonlinear Anal. TMA 67, 1947-1957 (2007). doi:10.1016/j.na.2006.08.030

11. Quirós, F, Rossi, JD: Non-simultaneous blow-up in a semilinear parabolic system. Z. Angew. Math. Phys. 52(2), $342-346$ (2001). doi:10.1007/PL00001549

12. Li, FC, Huang, SX, Xie, CH: Global existence and blow-up of solutions to a nonlocal reaction-diffusion system. Discrete Contin. Dyn. Syst. 9(6), 1519-1532 (2003). doi:10.3934/dcds.2003.9.1519

13. Ling, ZQ, Wang, ZJ: Simultaneous and non-simultaneous blow-up criteria of solutions for a diffusion system with weighted localized sources. J. Appl. Math. Comput. (2012). doi:10.1007/s12190-012-0570-z

14. Li, HL, Wang, MX: Critical exponents and lower bounds of blow-up rate for a reaction-diffusion system. Nonlinear Anal. TMA 63(8), 1083-1093 (2005). doi:10.1016/j.na.2005.05.037

15. Li, HL, Wang, MX: Blow-up behaviors for semilinear parabolic systems coupled in equations and boundary conditions. J. Math. Anal. Appl. 304(1), 96-114 (2005). doi:10.1016/j.jmaa.2004.09.020

16. Song, XF, Zheng, SN, Jiang, ZX: Blow-up analysis for a nonlinear diffusion system. Z. Angew. Math. Phys. 56, 1-10 (2005)

17. Deng, WB, Li, YX, Xie, CH: Blow-up and global existence for a nonlocal degenerate parabolic system. J. Math. Anal. Appl. 277, 199-217 (2003). doi:10.1016/S0022-247X(02)00533-4

18. Ling, ZQ, Wang, ZJ: Blow-up and global existence for a degenerate parabolic system with nonlocal sources. Discrete Dyn. Nat. Soc. 2012, Article ID 956564 (2012). doi:10.1155/2012/956564

19. Gidas, B, Ni, WM, Nirenberg, L: Symmetry of positive solutions of nonlinear elliptic equations in $R^{n}$. In: Nachbin, $L$ (ed.) Math. Anal. and Applications, Part A. Advances in Math. Suppl. Studies, vol. 7A, pp. 369-402. Academic Press, San Diego (1981)

20. Li, HL, Wang, MX: Global solutions and blow-up problems for a nonlinear degenerate parabolic system coupled via nonlocal sources. J. Math. Anal. Appl. 333(2), 984-1007 (2007). doi:10.1016/j.jmaa.2006.11.023

21. Friedman, A, Mcleod, B: Blow-up of positive solutions of semilinear heat equations. Indiana Univ. Math. J. 34(2), 425-447 (1985). doi:10.1512/iumj.1985.34.34025

doi:10.1186/1687-2770-2012-101

Cite this article as: Ling and Wang: Blow-up problems for a compressible reactive gas model. Boundary Value Problems 2012 2012:101. 